\title{
EFFECT OF PLASTIC STRAIN ON THE ELECTROCHEMICAL BEHAVIOR OF LEAN DUPLEX UNS S32304 STAINLESS STEEL*
}

\author{
Charles David ${ }^{1}$ \\ Fiona Ruel ${ }^{2}$ \\ Florent Krajcarz ${ }^{3}$ \\ Saghi Saedlou ${ }^{4}$ \\ Virginie Roche ${ }^{5}$ \\ Ricardo Nogueira ${ }^{6}$
}

\begin{abstract}
This work aimed at evaluating the effect of pre-strain of Lean Duplex UNS S32304 on anodic dissolution and hydrogen evolution reaction rates in acidic chloride media. Interrupted tensile testing was employed in order to generate different deformation levels. Potentiodynamic experiments allowed to detect a decrease on the cathodic and anodic reaction rates as a consequence of plastic deformation. A non-linear evolution of corrosion rates was observed, probably as a consequence of the evolution of specific dislocation structures in the material. Strain also caused a slight ennoblement of the ferritic phase, while austenite became a little less noble. These behaviors are analyzed in terms of residual stresses, characterized by X-ray diffraction.
\end{abstract}

Keywords: Corrosion; Strain; Duplex; Stainless.

\footnotetext{
PhD student, LEPMI - Université Grenoble-Alpes, APERAM Research Center, Isbergues, France. $P h D$, research engineer, APERAM Research Center, Isbergues, France. $P h D$, research engineer, APERAM Research Center, Isbergues, France. Engineer, Head of Solutions Dept, APERAM Research Center, Isbergues, France. $P h D$, researcher, LEPMI - Université Grenoble-Alpes, Grenoble, France. PhD, Professor, LEPMI - Université Grenoble-Alpes, Grenoble, France.
} 


\section{INTRODUCTION}

Austenitic-ferritic stainless steels, also known as Duplex (DSS), possess high mechanical properties and very good corrosion resistance. Among these steels, Lean Duplex, which contain very few or no molybdenum and less nickel than standard Duplex, are suitable for use in environments where standard Duplex would be overspecified from a corrosion resistance standpoint and thus represent an attractive technical and economic choice.

Given its applications, Lean DSS might undergo plastic strain during forming or inservice use. Although the corrosion behavior of these materials has been well studied, the effect of prior deformation on their corrosion resistance is not well known.

To this day, only a few works on the impact of strain of stainless steel on corrosion resistance have been carried out. The majority of them address pitting corrosion. $\mathrm{N}$. Renton and co-workers [1-2] observed that strain of Super Duplex induced an irregular evolution of pitting corrosion resistance in chloride medium, which they explain by the non-linear development of slip bands on the metal's surface. L. Peguet et al [3], while studying the effect of cold work on pitting corrosion of ferritic and austenitic stainless steels, observed an increase of the dissolution rate with cold work. Y. Huang et al [4] observed the same trend in a study on austenitic SS. That would be due to an increase of dislocation density in the material after plastic strain, and eventual changes on the corresponding surface morphology.

Even if pitting corrosion is a major concern for stainless steels, these materials are also employed for their resistance to stress corrosion cracking (SCC). Knowing if and how pre-strain would impact on SCC would be important. Such an impact has been observed by S. Yamazaki et al [5] in their study of the effect of prior deformation on the crack growth rates on a nickel-based alloy. The authors pointed out that the evolution of crack growth rate with cold work is not only related to the increasing of stress concentration at the crack tip, but also to the enhancement of the metal's oxidation kinetics. A high dislocation concentration near grain boundaries would result in a high oxidation rate.

The impact of prior deformation on oxidation rates but also on hydrogen evolution, which may cause embrittlement, has been addressed on different materials. Y. Cheng and co-workers [6-11] have concluded that strain would promote corrosion specially in the plastic region on high-strength steels [11]. That is in agreement with the mechanoelectrochemical interactions theory, which takes into account the activation of new dislocations in the material. In addition to that, their results show that, even if hydrogen evolution is also promoted by plastic strain, the impact on anodic dissolution is greater.

On the other hand, H. Krawiec et al, in their studies on different aluminum and titanium alloys, observe a predominant influence of strain on the cathodic reaction than on anodic dissolution [12-14]. The role of dislocations has been shown to be complex [14], since an increase of the number of dislocations enhances cathodic reaction up to a certain point, and then it decreases due to the formation of specific dislocation structures.

Dislocations have been proved to impact on dissolution of metal and hydrogen production in acidic media [15-20]. M. Sahal et al [15-16] observed that the dissolution rate of nickel in acidic medium depends on the dislocation density, and that taking into account the internal stresses generated by dislocation distribution is essential when correlating strain and anodic dissolution. Large et al's [17] studies confirmed it, and also proved that hydrostatic stresses play a role on dissolution. Regarding hydrogen 
production, El Alami et al [18-19] concluded that dislocations density and structures play a role on the adsorbing sites of the cathodic reaction on nickel.

Yet, strain is not suspected to impact on corrosion resistance only in terms of cathodic and anodic kinetics. Residual stress, which can be generated by straining, may cause corrosion potential shifts. Some authors have studied the effect of compressive and tensile stresses on corrosion resistance [20-22]. Globally, tensile stresses would shift the corrosion potential towards less noble values, whereas compressive stress would make materials nobler.

Briefly, for different materials, strain has been shown to impact on anodic dissolution and/or on the cathodic reaction, both of which are substantial parameters when considering Duplex stainless steels. Shifts of corrosion potentials due to residual stresses have also been reported.

This work aims at evaluating the effect of pre-strain of Lean Duplex UNS S32304 on anodic dissolution and hydrogen evolution reaction rates in acidic media. Furthermore, residual stresses are characterized by X-ray diffraction in order to evaluate their role on electrochemical behavior.

\section{MATERIAL AND METHODS}

An industrial grade of Lean Duplex stainless steel UNS S32304 produced by Aperam was used in this study. It was cold rolled down to a thickness of $1 \mathrm{~mm}$, annealed and pickled. The chemical content of main alloying elements is given in Table 1. Optical images acquired after chemical etching in Beraha's solution $(0,27 \mathrm{~g}$ potassium metabisulfite, $20 \mathrm{~mL} \mathrm{HCl}, 100 \mathrm{~mL}$ water) of the plate's section (Figure 1a) and surface (Figure 1b) allowed to evaluate the morphology of austenitic $(\gamma)$ and ferritic $(\alpha)$ zones. The average sectional band width was $1,34 \mu \mathrm{m}$ for ferrite and 1,07 $\mu \mathrm{m}$ for austenite. On the plate surface, images revealed a phase composition of $47,8 \% \alpha / 52,2 \% \mathrm{\gamma}$. A measurement of the magnetic phase by sigmameter revealed a larger ferritic ratio in the bulk, 53,9\% a/ 46,1\% $\mathrm{Y}$.

Table 1. Chemical composition of UNS S32304

\begin{tabular}{ccccccccc}
\hline C ppm & Mn \% & P \% & N ppm & Si \% & Ni \% & Cr \% & Cu \% & Mo \% \\
\hline 159 & 1.52 & 0.029 & 917 & 0.45 & 4.73 & 23.43 & 0.38 & 0.47 \\
\hline
\end{tabular}
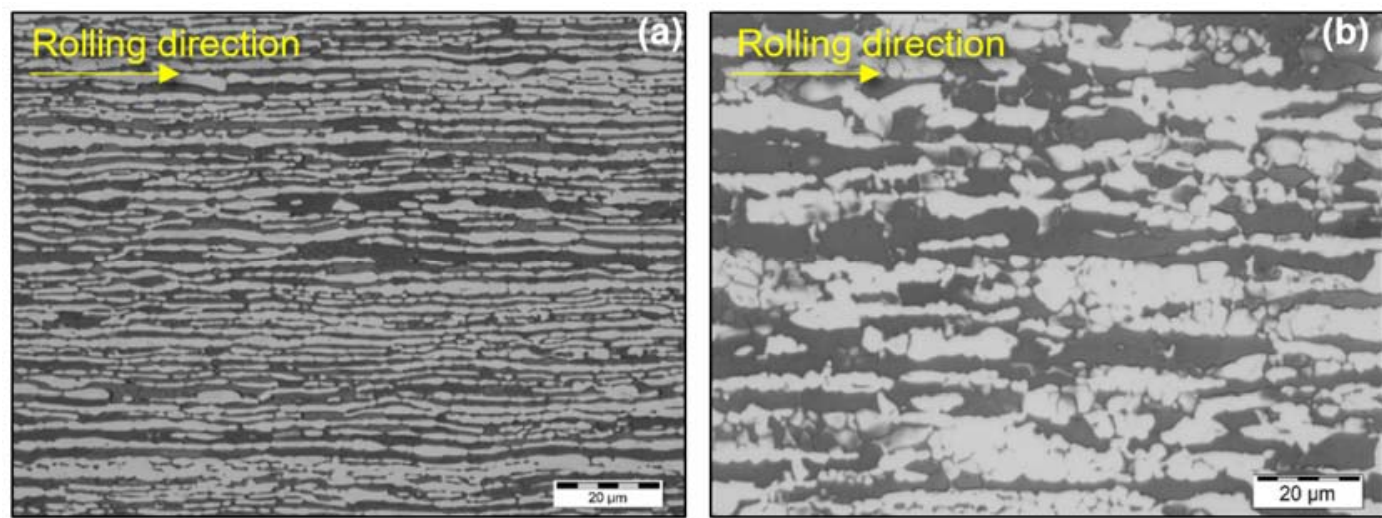

Figure 1. Micrographies of the plate's section (a) and of the surface (b) after etching 


\subsection{Interrupted tensile testing}

Due to technical constraints of a parallel ongoing study, tensile specimens were rectangular coupons of $100 \times 23 \mathrm{~mm}^{2}$. In order to ensure that the strain was uniaxial and homogenous in such a type of specimen, in-situ image correlation was carried out. For that purpose, specimens were covered with a white painting layer and then sprinkled with black painting so as to form the correlation dots (Figure 2).

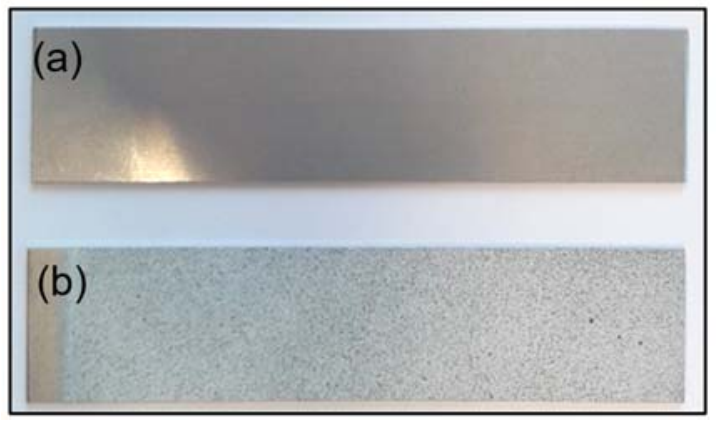

Figure 2. Tensile testing specimen for image correlation before (a) and after (b) painting application.

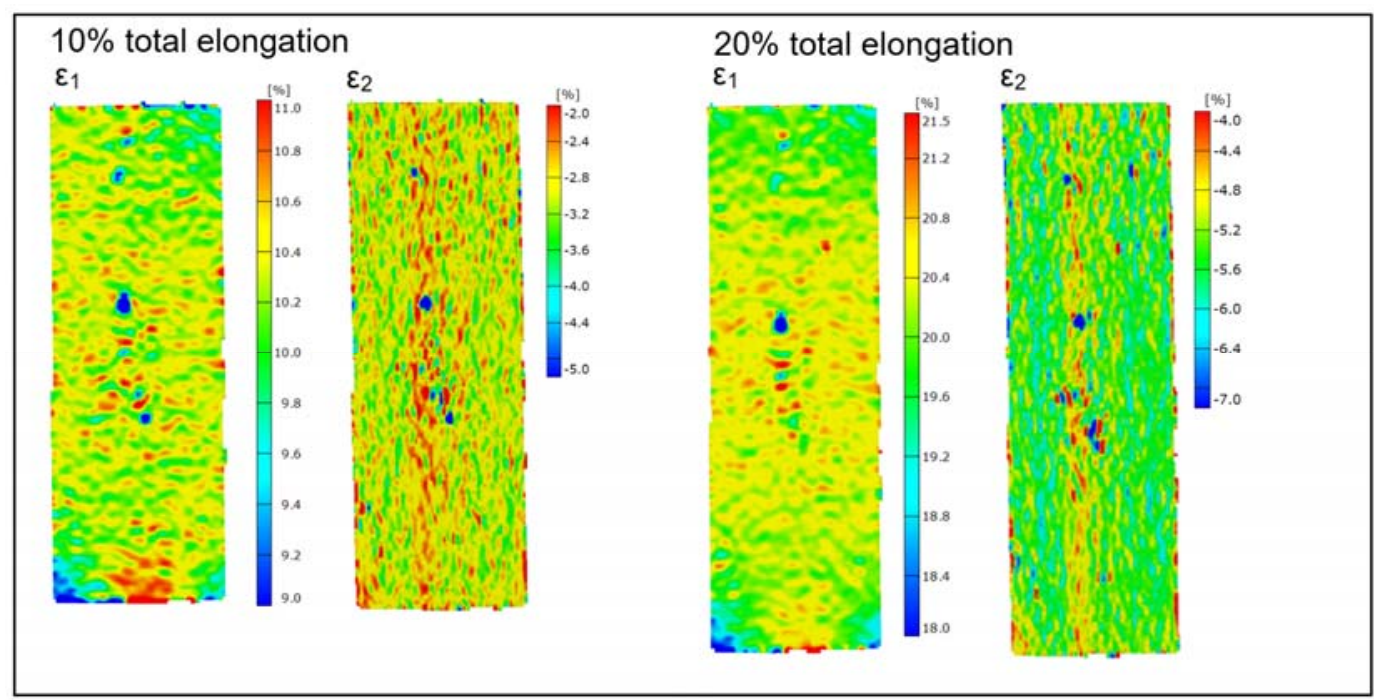

Figure 3. Strain maps by in-situ image correlation for $10 \%$ and $20 \%$ total elongation

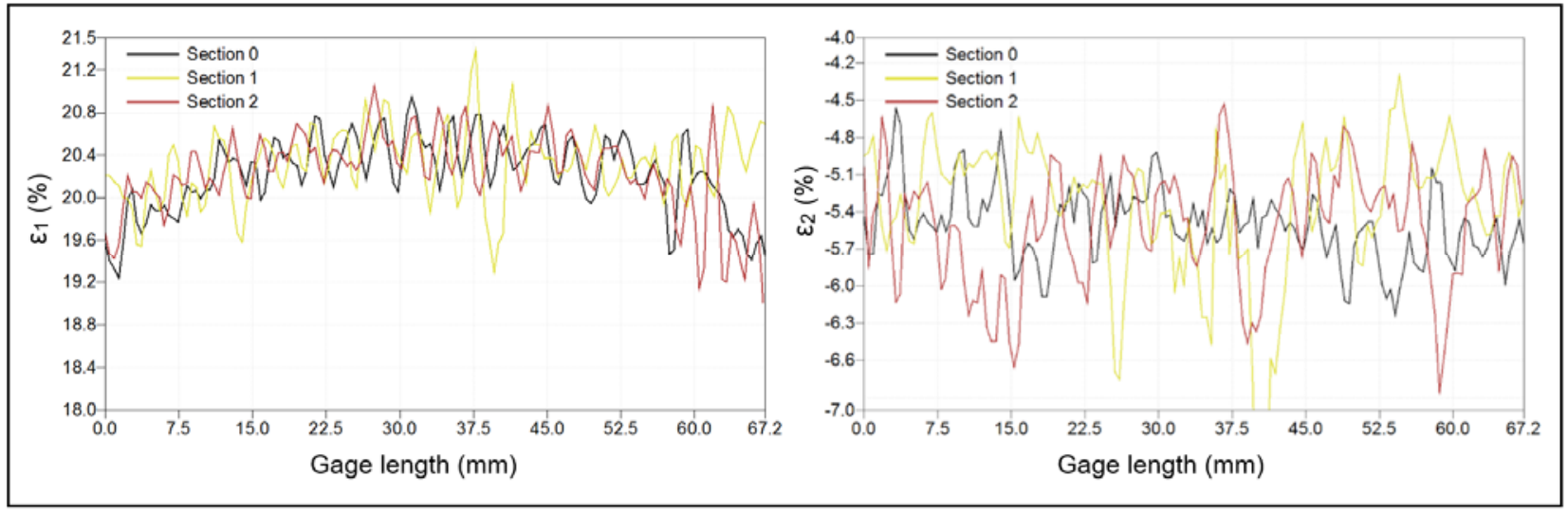

Figure 4. Strain profiles by in-situ image correlation for $20 \%$ total elongation 
The specimen was mounted on the tensile testing machine and centered so as the gage length was $60 \mathrm{~mm}$. A two-camera dispositive was placed in front of the gage section in order to capture images of the specimen during the test. The assembly configuration did not allow the use of an extensometer. The tensile test protocol started by a preload of $2 \mathrm{~N}$. Strain was controlled by the movement of the crosshead, with a constant rate of $0,5 \mathrm{~mm} / \mathrm{min}$ up to fracture. Images were captured every 22 seconds. For every image that was capture, the correlation software calculated both axial $\left(\varepsilon_{1}\right)$ and transverse $\left(\varepsilon_{2}\right)$ strains. Figure 3 shows the strain maps for $10 \%$ and $20 \%$ total elongation. In addition to that, longitudinal and transverse strain profiles were drawn on the axial direction of the specimen at $20 \%$ total elongation rate, at the center and near the edges of the specimen (Figure 4).

It was concluded that both axial and transverse strains were sufficiently homogeneous and uniaxial over a $45 \mathrm{~mm}$-length area in the center of the specimen until a $20 \%$ total elongation rate. The electrochemical experiments could therefore later be carried out on samples collected from that area.

For the fabrication of the pre-strained samples by tensile interrupted testing, the total elongation levels were: $0.5 \%, 1 \%, 3 \%, 5 \%, 10 \%$ and $20 \%$. The crosshead displacement needed for each one of these elongation levels was known based on the image correlation results, but this time an extensometer was used in order to know the final plastic strain. Specimens were strained using the same protocol that for image correlation. Once the desired total elongation for each specimen was reached, the crosshead's position was maintained for 5 minutes for deformation to accommodate, and then plastic strain was released down to zero load with a $-0,5 \mathrm{~mm} / \mathrm{min}$ crosshead displacement rate.

\subsection{Electrochemical experiments}

Two samples of approximately $21 \times 23 \mathrm{~mm}^{2}$ were collected from the center of each pre-strained specimen. They were then polished down to colloidal silica in order to avoid cold-working due to preparation. Surfaces were checked in SEM after polishing. Electrochemical experiments were carried out in a dual-vessel three-electrode assembly thermostated at $23^{\circ} \mathrm{C}$, using an $18 \mathrm{~mm}$ O-ring working electrode. The configuration of the system allowed purging the electrochemical media with a 20/80 $\mathrm{H}_{2} / \mathrm{N}_{2}$ mixture for 1 hour before each experiment without any contact between the media and the sample during purging. For each sample, the electrochemical protocol started with a 5-minute potentiostatic current measurement at $-0,6$ V/SCE in a 0.07 $\mathrm{mol} / \mathrm{L} \mathrm{H}_{2} \mathrm{SO}_{4}+200 \mathrm{~g} / \mathrm{L} \mathrm{NaCl}$ solution. Then, polarization curves were immediately drawn, starting at $-0,6 \mathrm{~V} / \mathrm{SCE}$ towards anodic potentials with a $20 \mathrm{mV} / \mathrm{min}$ scanning rate till $-0,1 \mathrm{~V} / \mathrm{SCE}$.

\subsection{Internal stresses measurement}

Internal stress measurements were carried out on an X-ray diffractometer using the $\sin ^{2} \Psi$ method along the axial direction of tensile straining. X-rays were generated by a cobalt tube. The peak at $89.173^{\circ}$ was chosen for the study of austenite, and at $99.374^{\circ}$ for ferrite, with a scanning range of $6^{\circ}$ and a step of $0.06^{\circ}$. 


\section{RESULTS AND DISCUSSION}

\subsection{Interrupted tensile testing}

Engineering stress-strain curves plotted from the extensometer data (Figure 5) allowed to know the final plastic strain for each pre-strain level (Table 2). It can be noted from the plots that curves are quite stackable, indicating the same strain path. Henceforth, given that the aim of the present study is to correlate plastic strain and corrosion, only the final plastic strain will be used. For practical reasons, numbers will be written under percentage form: $0.4 \%, 0.9 \%, 3.3 \%, 4.3 \%, 9.4 \%, 19.3 \%$.

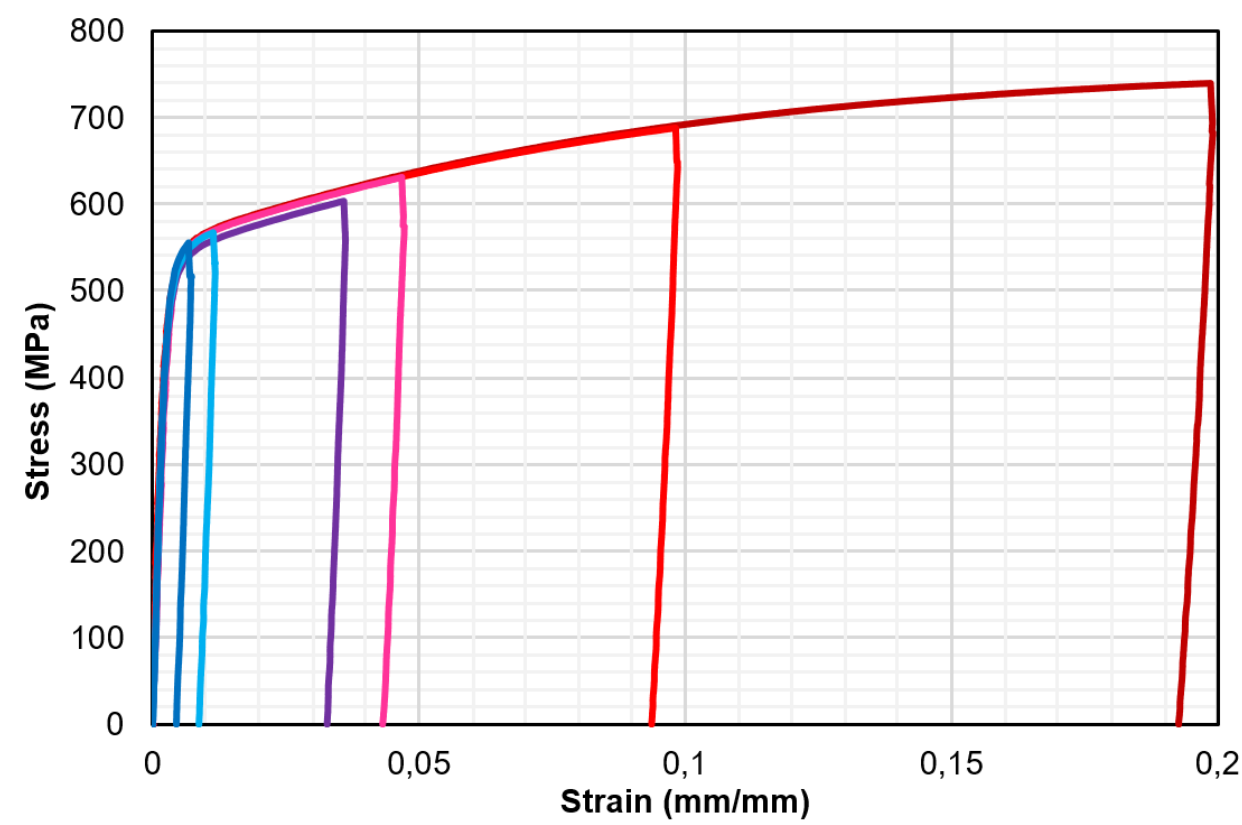

Figure 5. Engineering stress-strain curves of interrupted tensile testing.

Table 2. Targeted total elongation and corresponding plastic strain

\begin{tabular}{cc}
\hline Targeted total elongation & $\boldsymbol{\varepsilon}_{\mathbf{p}}$ (extensometer) \\
\hline $0.5 \%$ & 0.004 \\
\hline $1 \%$ & 0.009 \\
\hline $3 \%$ & 0.033 \\
\hline $5 \%$ & 0.043 \\
\hline $10 \%$ & 0.094 \\
\hline $20 \%$ & 0.193 \\
\hline
\end{tabular}

\subsection{Effect of plastic strain on anodic dissolution and hydrogen evolution}

Polarization curves show two activity peaks (Figure 6), as it was expected for a DSS. It has already been demonstrated that the peak at more negative potentials corresponds to ferrite, and the more anodic one to austenite [23]. A distinction can be observed between low strained (unstrained to $0.9 \%$ ), and strongly strained $(3.3 \%$ to $19.3 \%$ ) samples, especially in terms of cathodic currents and ferrite's activity peak. The same curves are drawn in Figure 7, but currents are expressed in logarithmic scale. On this graph, the reduction of both cathodic and anodic rates with pre-strain 
are highlighted. These current reductions mean that, for a given cathodic potential, hydrogen production decreases with pre-strain. For a given anodic potential, ferrite's oxidation also decreases with strain.

Moreover, strain induces a slight shift of the corrosion potential $\left(E_{c o r r}\right)$ towards more positive potentials. Given that at the corrosion potential the oxidation mainly concerns ferrite, this phase is believed to have become nobler with strain. Even if austenite is not the phase being predominantly corroded in this medium, a negative shift of the minima between ferrite's and austenite's activity peaks $\left(E_{\min }\right)$ is observed, suggesting that the austenitic phase might have become less noble.

In order to better evaluate the evolution of corrosion rates $\left(\mathrm{J}_{\text {corr }}\right)$, they were calculated using the cathodic Tafel rights and plotted against plastic strain on Figure 9a. Corrosion rate first increases with strain for low pre-strain levels, and then decreases and stabilizes for higher strains. That non-linear observation corresponds well to previous studies and could be explained based on the role of dislocations. As raised by $\mathrm{H}$. Krawiec et al and M. Sahal et al [12-16], the increase of the number of dislocations due to plastic strain enhances cathodic reaction to a certain level, and then slows it down due to the formation of specific dislocation structures, which hinders adsorption processes. L. Peguet and Y. Huang et al $[3,4]$ also mentioned an increase of dissolution rates due to an increase of dislocations density.

Figures $9 \mathrm{~b}$ and $9 \mathrm{c}$ show the variations of the corrosion potential and the potential at the beginning of the activity peak of austenite compared to the potentials for the unstrained sample. Although the interpretation of the variation for austenite might be complex, for ferrite (corrosion potential) it appears to correspond to the evolution of the corrosion rates, except for low strains. It is possible that the $\mathrm{E}_{\text {corr }}$ shift towards more noble potentials is globally a result of a more marked effect of pre-strain on the oxidation reaction of ferrite than on cathodic reduction, as found by X. Ly et al [11].

Residual stress measurements on unstrained and $19.3 \%$ pre-strained samples are given on Table 3. Austenite is under tensile stress before straining, and ferrite under compressive stress. After straining, the character of internal stresses in each phase does not change, except for its magnitude that increases. That could also explain the shifts on $E_{\text {corr }}$ and $E_{\min }$, since compressive stresses are supposed to make materials nobler, and inversely for tensile stresses [20-22]. In Duplex steels, it can be supposed that, given that the two phases do not have the same mechanical behavior, after prestrain and elastic relaxation the softer phase would retract more than the harder phase, increasing compressive residual stresses in the first one and tensile in the second.

Further studies should allow to evaluate the contribution of dislocations and also to better understand the evolution of cathodic and anodic behaviors separately after straining. 


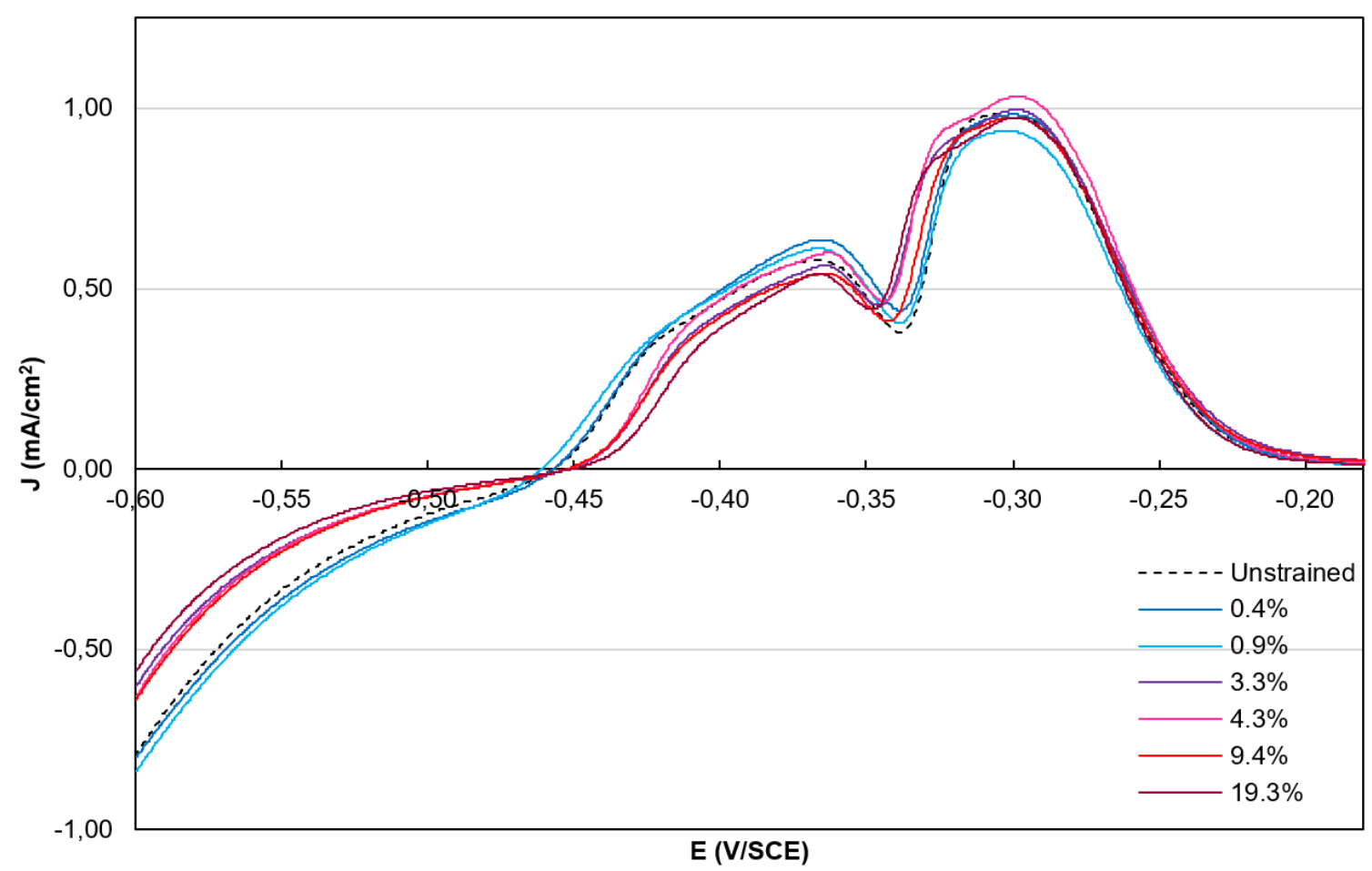

Figure 6. Polarization curves of unstrained and pre-strained Lean Duplex UNS 32304 in $0.07 \mathrm{M} \mathrm{H}_{2} \mathrm{SO}_{4}+200 \mathrm{~g} / \mathrm{L} \mathrm{NaCl}$.

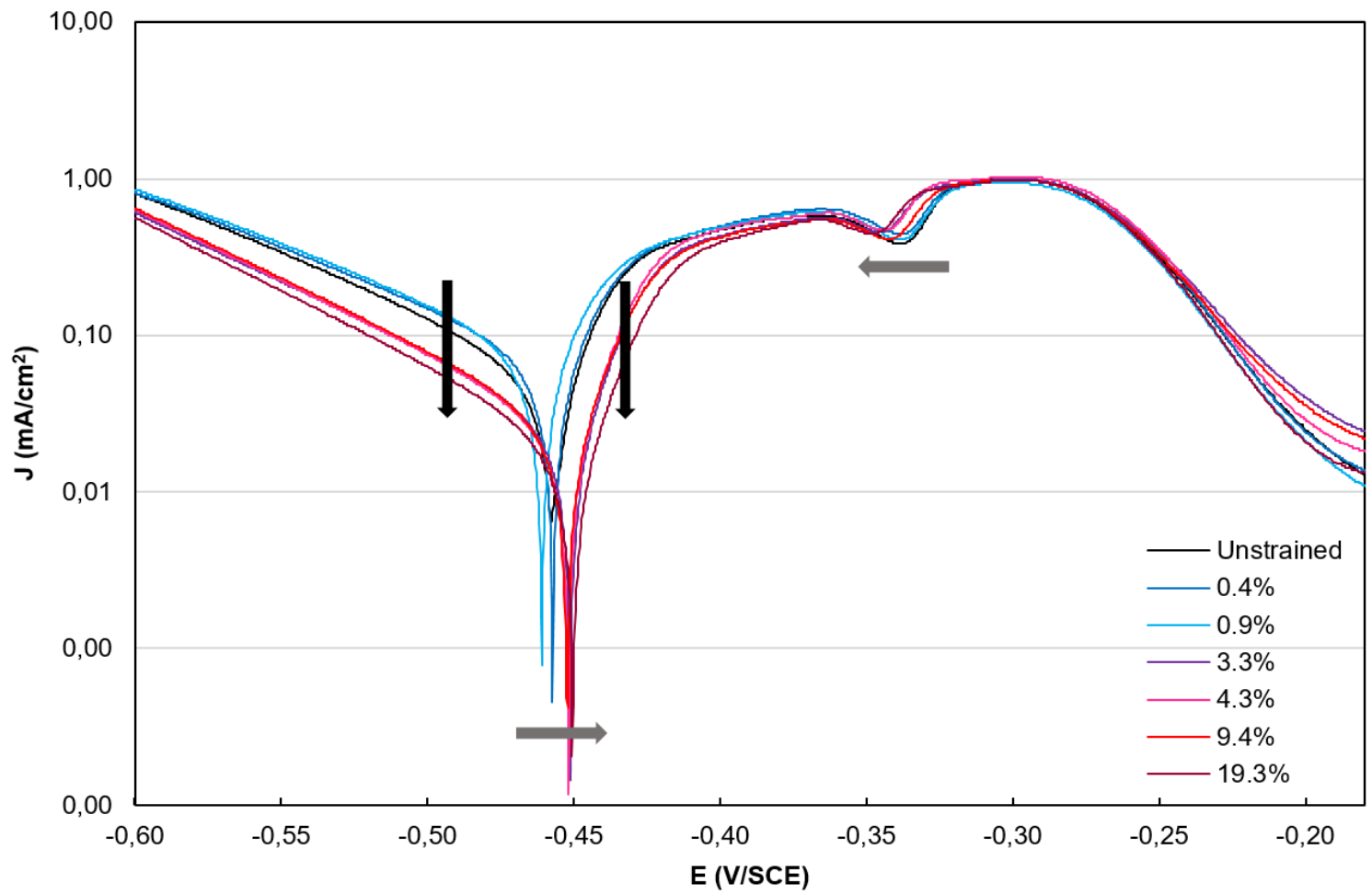

Figure 7. Log-scale polarization curves of unstrained and pre-strained Lean Duplex UNS 32304 in $0.07 \mathrm{M} \mathrm{H}_{2} \mathrm{SO}_{4}+200 \mathrm{~g} / \mathrm{L} \mathrm{NaCl}$ - evolution of currents and potentials. 



Axial plastic strain (\%)

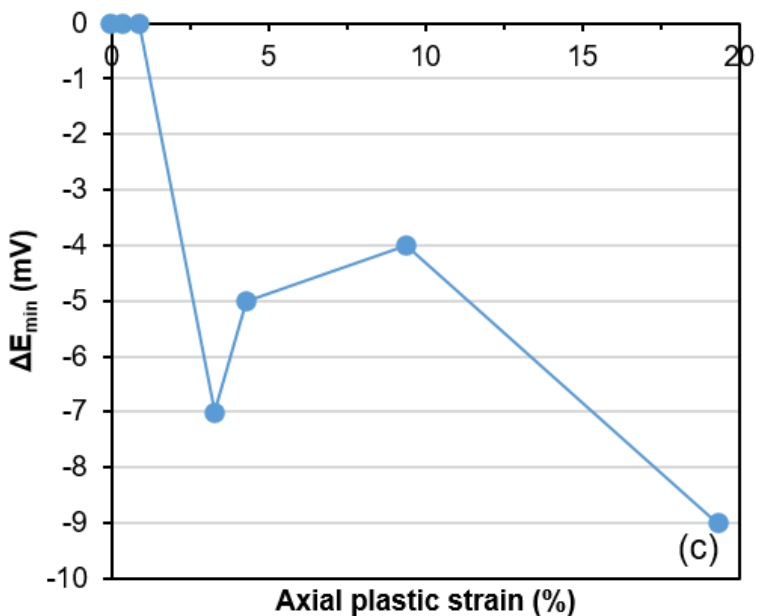

Figure 9. Evolution of the corrosion rates (a), corrosion potentials (b) and $E_{\min }(c)$ as a function of pre-strain.

Table 3. Residual stresses as a function of pre-strain level along axial direction

\begin{tabular}{cc|c}
\multirow{2}{*}{ Pre-strain level } & \multicolumn{2}{c}{ Residual stress (MPa) } \\
\cline { 2 - 3 } & Austenite & Ferrite \\
\hline Unstrained & 38.3 & -95.0 \\
\hline $19.3 \%$ & 72.2 & -169.7 \\
\hline
\end{tabular}

\section{CONCLUSION}

A protocol for evaluation of the effect of plastic strain obtained by interrupted tensile testing on the electrochemical behavior of Duplex stainless steels has been set up.

Potentiodynamic experiments on seven pre-strain levels allowed to detect a difference on the cathodic and anodic reaction rates as a consequence of plastic deformation. First, corrosion rates increase with strain for the low pre-strain levels. This would be due to the development of new dislocations in the material. For higher deformation, corrosion rates decrease and then stabilize, probably as a consequence of the evolution of specific dislocation structures that hinder adsorption processes.

A slight ennoblement of ferrite has been observed for plastic strain levels going from $3.3 \%$ to $19.3 \%$. The opposite behavior was noted for austenite. If that trend is 
confirmed, it could be due both to the effect of strain on the anodic dissolution reaction and to the residual stress state in the material.

\section{REFERENCES}

[1] Elhoud AM, Renton NC, Deans WF. The effect of manufacturing variables on the corrosion resistance of a super duplex stainless steel. The International Journal of Advanced Manufacturing Technology. 2011 Feb;52(5-8):451-61.

[2] Renton NC, Elhoud AM, Deans WF. Effect of Plastic Deformation on the Corrosion Behavior of a Super-Duplex Stainless Steel. Journal of Materials Engineering and Performance. 2011 Apr;20(3):436-44.

[3] Peguet L, Malki B, Baroux B. Influence of cold working on the pitting corrosion resistance of stainless steels. Corrosion Science. 2007 Apr;49(4):1933-48.

[4] Huang Y, Xuan F-Z, Tu S-T, Itoh T. Effects of hydrogen and surface dislocation on active dissolution of deformed 304 austenitic stainless steel in acid chloride solution. Materials Science and Engineering: A. 2011 Jan;528(3):1882-8.

[5] Yamazaki S, Lu Z, Ito Y, Takeda Y, Shoji T. The effect of prior deformation on stress corrosion cracking growth rates of Alloy 600 materials in a simulated pressurized water reactor primary water. Corrosion Science. 2008 Mar;50(3):835-46.

[6] Cheng Y. Analysis of electrochemical hydrogen permeation through X-65 pipeline steel and its implications on pipeline stress corrosion cracking. International Journal of Hydrogen Energy. 2007 Jun;32(9):1269-76.

[7] Tang X, Cheng YF. Micro-electrochemical characterization of the effect of applied stress on local anodic dissolution behavior of pipeline steel under near-neutral $\mathrm{pH}$ condition. Electrochimica Acta. 2009 Feb;54(5):1499-505.

[8] Zhang GA, Cheng YF. Micro-electrochemical characterization of corrosion of precracked $\mathrm{X} 70$ pipeline steel in a concentrated carbonate/bicarbonate solution. Corrosion Science. 2010 Mar;52(3):960-8.

[9] Xue HB, Cheng YF. Electrochemical corrosion behavior of X80 pipeline steel in a near-neutral pH solution. Materials and Corrosion. 2010 Sep;61(9):756-61.

[10] Xu LY, Cheng YF. An experimental investigation of corrosion of X100 pipeline steel under uniaxial elastic stress in a near-neutral pH solution. Corrosion Science. 2012 Jun;59:103-9.

[11] Xu LY, Cheng YF. Corrosion of X100 pipeline steel under plastic strain in a neutral pH bicarbonate solution. Corrosion Science. 2012 Nov;64:145-52.

[12] Krawiec H, Szklarz Z, Vignal V. Influence of applied strain on the microstructural corrosion of AIMg2 as-cast aluminium alloy in sodium chloride solution. Corrosion Science. 2012 Dec;65:387-96.

[13] Krawiec H, Vignal V, Schwarzenboeck E, Banas J. Role of plastic deformation and microstructure in the micro-electrochemical behaviour of Ti-6Al-4V in sodium chloride solution. Electrochimica Acta. 2013 Aug;104:400-6.

[14] Krawiec H, Vignal V, Loch J, Erazmus-Vignal P. Influence of plastic deformation on the microstructure and corrosion behaviour of $\mathrm{Ti}-10 \mathrm{Mo}-4 \mathrm{Zr}$ and $\mathrm{Ti}-6 \mathrm{Al}-4 \mathrm{~V}$ alloys in the Ringer's solution at $37^{\circ} \mathrm{C}$. Corrosion Science. $2015 \mathrm{Jul} ; 96: 160-70$.

[15] Sahal M, Creus J, Sabot R, Feaugas X. Consequences of plastic strain on the dissolution process of polycrystalline nickel in $\mathrm{H} 2 \mathrm{SO} 4$ solution. Scripta Materialia. 2004 Nov;51(9):869-73.

[16] Sahal M, Creus J, Sabot R, Feaugas X. The effects of dislocation patterns on the dissolution process of polycrystalline nickel. Acta Materialia. 2006 May;54(8):215767. 
[17] Large D, Sabot R, Feaugas X. Influence of stress-strain field on the dissolution process of polycrystalline nickel in $\mathrm{H} 2 \mathrm{SO} 4$ solution: An original in situ method. Electrochimica Acta. 2007 Oct;52(27):7746-53.

[18] El Alami H, Creus J, Feaugas X. Influence of the plastic strain on the hydrogen evolution reaction on polycrystalline nickel electrodes in H2S04. Electrochimica Acta. 2006 Jun;51(22):4716-27.

[19] El Alami H, Creus J, Feaugas X. Thermodynamic parameters evolution versus plastic strain during HER on nickel in sulphuric acid. Electrochimica Acta. 2007 Mar;52(12):4004-14.

[20] Zhang Y, Poursaee A. Passivation and Corrosion Behavior of Carbon Steel in Simulated Concrete Pore Solution under Tensile and Compressive Stresses. Journal of Materials in Civil Engineering. 2015 Aug;27(8):04014234.

[21] Navai F. Effects of tensile and compressive stresses on the passive layers formed on a type 302 stainless steel in a normal sulphuric acid bath. Journal of materials science. 1995;30(5):1166-72.

[22] Navai F. Electrochemical behaviour of a type 302 stainless steel in a stress field. Journal of materials science. 2000;35(23):5921-5.

[23] Ruel F, Tite D, Gaugain A, Saedlou S, Wolski K. On the Depassivation Mechanism of Lean Duplex Stainless Steels and the Influence of the Partitioning of the Alloying Elements. Corrosion. 2014 Jun;70(6):636-42. 